\title{
Bound states of two dyons and two fermions
}

\author{
P.C. Pant, V.P.Pandey and B.S. Rajput ${ }^{1^{*}}$ \\ Department of Physics, Kumaun University, Nainital-263002, India
}

\begin{abstract}
Investigating the bound state of a system consisting of two dyons and two fermions it has been demonstrated that due to the presence of magnetic charge on dyon energy eigenvalues and eigen function for this system are largely modified from the energy eigenvalues and eigenfunction of the usual Helium atom problem of quantum electrodynamics . Bohr radius for this system is shown to be very small in comparison to atomic Bohr radius implying the strongly interacting behavior of dyons. It has also been shown that the repulsive force between dyons is greater than the repulsive force between the nucleus of Helium molecule implying the small possibility of forming bound states.
\end{abstract}

\section{INTRODUCTION}

System of magnetic monopoles ${ }^{1}$ has dominated the theories of particle physics during the last two decades. Many attempts have been made by different authors ${ }^{2}$ to construct a self-consistent quantum field theory revealing their general properties. Despite the enormous potential importance of these particles, in connection with their role in catalyzing proton-decay ${ }^{3}$, magnetic condensation of vacuum ${ }^{4}$, C-P violation, quark confinement mechanism ${ }^{5}$ and the unification of fundamental interactions ${ }^{6}$, most of the theories to describe them suffered from many paradoxes such as Dirac veto ${ }^{1}$ and wrong connection between spin and statistics ${ }^{7}$. Two separate approaches have revived interest in the existence of such particles. The first one is the idea of 't Hooft and Polyakov ${ }^{8,9}$ who demonstrated separately the existence of these particles from the general idea of unification of fundamental interactions and showed that all such grand

\footnotetext{
${ }^{*}$ Corresponding author

${ }^{1}$ Chariman, U.P. State Council for Higher Education, Lucknow (Infia).

Vice-chancellor, Kumaun University, Nainital -263001 (India)
} 
unified theories necessarily contain magnetic monopoles. In the second approach Witten ${ }^{10}$ has demonstrated that some of the paradoxes of the theories of magnetic monopoles can be resolved by considering electric and magnetic charges on the same particle called dyon. In addition to this he showed that monopoles are necessarily dyons. In order to investigate the quantal properties of these particles for their experimental verification it became necessary to construct a transparent Quantum field theory of these particles and to investigate their bound states. Keeping these facts in view we have developed ${ }^{11} \mathrm{a}$ self-consistent quantum field theory of generalized electromagnetic field associated with these particles by using two-four potentials to avoid the use of arbitrary string variables ${ }^{1}$ and assuming the generalized charge, generalized four-current and generalized fourpotential associated with these particles as complex quantities with their real and imaginary parts as electric and magnetic constituents. We have also undertaken the study of Pauli equation for fermion-dyon dynamics ${ }^{12,13,14}$ by ad hoc introduction of spin in the system to get bound state solutions. Extending this work in the present paper we have undertaken the study of bound state of two dyons and two fermions to obtain their bound state energy energyvalues and eigenfunctions. It has also been demonstrated that this system becomes very complicated and it is very difficult to get exact solutions. However, some meaningful solutions have been obtained under certain approximations, which are largely modified from the usual bound state solutions of Helium atom due to presence of magnetic charge.

\section{FIELD ASSOCIATED WITH GENERALIZED CHARGES}

Let us introduce the generalized charge $\mathrm{q}$ as a complex quantity

$$
q=e-i g
$$

where $e$ and $g$ are electric and magnetic charges on electromagnetic dyon. Assuming the generalized field $\Psi(x)$, generalized four-current $\left\{J_{\mu}\right\}$ and generalized four-potential $\left\{V_{\mu}\right\}$ as complex quantities in the similar manner, we can write the equation of motion of generalized charge in the generalized electromagnetic field in the following form ${ }^{[6]}$;

$$
M x=\operatorname{Re}\left[q\left\{\vec{\psi}^{*}-i\left(\vec{v} \times \vec{\psi}^{*}\right)\right\}\right]
$$

where Re denotes the real part, $\vec{v}$ in the velocity of the particle and $\psi(x)$ is the generalized field defined as

$$
\vec{\psi}(x)=\vec{E}-i \vec{H}
$$

in terms of electric and magnetic fields $(\vec{E}$ and $\vec{H})$.We can also write equation (2.2) in the following way

$$
M x=e\{\vec{E}+(\vec{v} \times \vec{H})\}+g\{\vec{H}-(\vec{v} \times \vec{H})\}
$$


by using equation (2.3). Equation (2.4) is the usual Lorenz equation of motion for electromagnetic dyon. Gauge invariant and rotationally symmetric angular momentum operator of the generalized charge $\mathrm{q}_{\mathrm{j}}$ moving in the field of another dyon $\mathrm{q}_{\mathrm{k}}$ has been obtained in the following form [5,6];

$$
\vec{J}=\vec{r} \times\left\{\vec{P}-\operatorname{Im}\left(q_{j} q_{k}^{*}\right) \vec{V}\right\}+\operatorname{Im}\left(q_{j} \cdot q_{k}^{*}\right) \frac{\vec{r}}{r}
$$

where $\vec{V}$ is the spatial part of the generalized four-potential $\{V \mu\}$. The gauge invariant linear momentum operator of the $\mathrm{j}^{\text {th }}$ generalized charge $\mathrm{q}_{\mathrm{j}}$ interacting with the field of $\mathrm{k}^{\text {th }}$ generalized charge $\mathrm{q}_{\mathrm{k}}$ has the following form;

$$
\vec{\pi}=\vec{P}-\operatorname{Im}\left(q_{j} q_{k}^{*}\right) \vec{V}
$$

Equations (2.5) and (2.6) lead to the following expression of gauge invariant and rotationally symmetric Hamiltonian operator for interacting generalized charges $q_{j}$ and $\mathrm{q}_{\mathrm{k}}^{*}$

$$
\mathrm{H}=\frac{\vec{\pi}^{2}}{2 m}-\frac{\operatorname{Re}\left(q_{j} q_{k}^{*}\right)}{r}+\frac{\operatorname{Im}\left(q_{j} q_{k}^{*}\right)^{2}}{2 M r^{2}}
$$

where the first term corresponds to kinetic energy while the second and third terms give the interaction potential energy of generalized charges i.e.

$$
V(r)=-\frac{\operatorname{Re}\left(q_{j} q_{k}^{*}\right)}{r}+\frac{\operatorname{Im}\left(q_{j} q_{k}^{*}\right)^{2}}{2 M r^{2}}
$$

Equation (2.5) directly gives the scalar

$$
\frac{\vec{r} \cdot \vec{j}}{r}=\operatorname{Im}\left(q_{j} q_{k}^{*}\right)
$$

which commutes with all the observables and shows that in equation (2.5) there is a residual angular momentum

$$
\vec{J}_{\text {res }}=\operatorname{Im}\left(q_{j} q_{k}^{*}\right) \frac{\vec{r}}{r}
$$

carried by the generalized field of generalized charge besides the orbital and spin angular momentum of each particle. This angular momentum can be identified as Wilson type of angular momentum ${ }^{6}$.It arises due to the rotation of the system of two dyons in the charge space around the line joining them. It may also be described as extra spin of the system which cannot be associated with either particle alone. Furthermore, if the generalized angular momentum given by equation (2.6) is quantized along the line joining generalized charges $q_{j}$ and $q_{k}$, we obtain the following quantization condition for generalized charges;

$$
\operatorname{Im}\left(q_{j} q_{k}^{*}\right)=n
$$


where $n$ is an integer. It reduces to the following chirality quantization condition for electrodyons;

$$
\operatorname{Im}\left(q_{j} q_{k}^{*}\right)=\mu_{j k}=e_{k} g_{j}-g_{k} e_{j}=0, \pm 1, \pm 2, \ldots
$$

Similarly, the $\operatorname{Re}\left(q_{j} q_{k}^{*}\right)$ may be written as

$$
\operatorname{Re}\left(q_{j} q_{k}^{*}\right)=\alpha_{j k}=e_{j} e_{k}+g_{j} g_{k}
$$

\section{BOUND STATES OF TWO DYONS AND TWO FERMIONS}

In order to study the interaction between two dyons and two fermions we assume that dyon move more slowly than the fermion as dyons are a few thousands times heavier than fermions. As such it seems reasonable to assume the dyons of this system are at rest in the zeroth approximation and subsequently to consider the motion of dyons in the higher orders of approximation using perturbation theory. Therefore, in the zeroth approximation, where the mass of two dyons is considered to be infinitely large, the problem of finding the stationary states of the system reduces to solving the Schrodinger equation;

$$
\left[\hat{H}_{0}-\varepsilon_{n}(R)\right] \phi_{n}(R, r)=0
$$

for fixed values of coordinate $R$ of the heavy particle. The index $\mathrm{n}$ determines all quantum numbers characterizing the stationary states. For each state, corresponding to a well-defined value of $n$, the energy of the system $\varepsilon_{n}(r)$ and the wavefunction $\phi_{n}(R, r)$ depend on the heavy particle coordinate $R$ as parameter. The function $\phi_{n}(R, r)$ thus characterizes

a state of motion of light fermion for fixed values of co-ordinate or for infinitely slow (adiabatic) changes in $R$. We can write the Hamiltonian for such system as follows

$$
\hat{H}_{0}=-\frac{1}{2 \mu}\left[\hat{\pi}_{1}^{2}+\hat{\pi}_{2}^{2}\right]+V(r)
$$

where $\hat{\pi}$, given by equation (2.6) is the momentum of a dyon moving in the generalized electromagnetic field of another dyon and $\mu_{i j}$ is the magnetic coupling parameter defined by equation (2.12). Second term in the Hamiltonian is the interaction potential term and its value is given as 


$$
\begin{aligned}
V(r)= & -\left[\frac{e_{1} e_{2}}{r_{12}}-\frac{e_{i} e_{1}}{r_{A_{1}}}-\frac{e_{2} e_{i}}{r_{A_{2}}}-\frac{e_{1} e_{j}}{r_{B_{1}}}-\frac{e_{2} e_{j}}{r_{B_{2}}}+\frac{\left(e_{1} g_{i}\right)^{2}}{2 m r_{A_{1}}^{2}}+\frac{\left(e_{2} g_{j}\right)^{2}}{2 m r_{B_{2}}^{2}}+\frac{\left(e_{1} g_{j}\right)^{2}}{2 m r_{B_{1}}^{2}}\right. \\
& \left.+\frac{\left(e_{2} g_{i}\right)^{2}}{2 m r_{A_{2}}^{2}}+\frac{\operatorname{Re}\left(e_{i}-i g_{i}\right)\left(e_{j}+i g_{j}\right)}{R}-\frac{\operatorname{Im}\left(e_{i}-i g_{i}\right)\left(e_{j}+i g_{j}\right)}{2 M R^{2}}\right] \ldots \text { (3.3) }
\end{aligned}
$$

In this equation $\mathrm{M}$ is the mass of dyon, $e_{1}$ and $e_{2}$ are the charges of fermions while $e_{\mathrm{i}}, e_{\mathrm{j}}$, and $g_{\mathrm{i}}, g_{\mathrm{j}}$ are the electric and magnetic charges on the dyons $A$ and $B$ respectively. The generalized charge $q$ is defined as

$$
q=e-i g
$$

The complicated system, described by eqn.(3.2), can be simplified when we make the following assumptions

$$
\left|e_{1}\right|=\left|e_{2}\right| \text { and }\left|q_{i}\right|=\left|q_{j}\right| i . e .\left|e_{i}\right|=\left|e_{j}\right| \text { and }\left|g_{i}\right|=\left|g_{j}\right|
$$

with these assumptions we obtain

$$
\left.\begin{array}{l}
\operatorname{Re}\left(e_{i}-i g_{i}\right)\left(e_{i}+i g_{i}\right)=e^{2}+g^{2} \\
\text { and } \\
\operatorname{Im}\left(e_{i}-i g_{i}\right)\left(e_{i}+i g_{i}\right)=0
\end{array}\right\}
$$

Substituting these values from equations (3.5) and (3.5a) in to equation (3.1), we get

$$
\begin{aligned}
\hat{H}_{0}= & -\frac{\hbar^{2}}{2 \mu}\left(\hat{\nabla}_{1}^{2}+\hat{\nabla}_{2}^{2}\right)-\left[\frac{e^{2}}{r_{A_{1}}}-\frac{e^{2}}{r_{12}}-\frac{e^{2}}{r_{B_{1}}}-\frac{e^{2}}{r_{B_{2}}}-\frac{e^{2}}{r_{A_{2}}}\right. \\
& \left.+\frac{(e g)^{2}}{2 m r_{A_{1}}^{2}}+\frac{(e g)^{2}}{2 m r_{B_{2}}^{2}}+\frac{(e g)^{2}}{2 m r_{A_{2}}^{2}}+\frac{(e g)^{2}}{2 m r_{B_{2}}^{2}}+\frac{e^{2}+g^{2}}{R}\right]
\end{aligned}
$$

We can solve this bound state problem in zeroth approximation by HeitlerLondon method after modifying it for the wavefunctions of isolated dyon-fermion system. The energy of the system in the first approximation is determined by the average value of the operator $\hat{H}_{0}$ in the state corresponding to the zeroth approximation wavefunctions. The wavefunction of two dyons-two fermion bound state is constructed from the wavefunction of the 1-S ground state of the dyon-fermion ${ }^{8}$ system. However, the probability of forming this bound state will be very small as the repulsive force between dyons will be large as the distance of closest approach will be very much smaller than Bohr radius $a_{0}$. When choosing the zeroth approximation we must take in to account the symmetry of the wavefunction due to identical fermions. 
In order to obtain the wavefunction for 1-S ground state of the bound state of dyon-'A' and fermion '1', let us start with the following Schrodinger equation;

$$
\hat{H} \psi=E \psi
$$

where $\hat{H}$ is given by ${ }^{[8]}$

$$
\hat{H}=\frac{\hat{\pi}^{2}}{2 m}-\frac{k}{r_{A_{1}}}+\frac{p^{2}}{2 m r_{A_{1}}^{2}}
$$

where $k$ and $p$ are constants defined as

$$
k=e_{i} e_{j} \quad \text { and } \quad p=e_{i} g_{j}
$$

and $\hat{\pi}$ is the gauge-invariant linear momentum operator

$$
\hat{\pi}^{2}=\left(\vec{P}-e_{i} g_{j} \vec{V}^{T}\right)
$$

The first term in equation (3.8) is the kinetic energy term, while the second and third terms give the interaction potential energy of the system. For this system, the symmetric and gauge-invariant angular momentum of the fermion with charge $e_{\mathrm{i}}$ moving in the field of an electromagnetic dyon $q_{\mathrm{j}}$ is given as

$$
\vec{L}=\vec{r}_{A_{1}} \times\left(\hat{P}-e_{i} g_{j} \vec{V}^{T}\right)+\frac{e_{i} g_{j} \vec{r}_{A_{1}}}{\left|\vec{r}_{A_{1}}\right|}
$$

where $\vec{V}^{T}$ is the transverse generalized potential of the field associated with $\mathrm{j}^{\text {th }}$ dyon. The wave function $\psi$ of equation (3.7) can be written in the following form

$$
\psi=\frac{U(r)}{r_{A_{1}}} Y_{e g, l^{\prime}, m^{\prime}}
$$
where $Y_{e g, l^{\prime} m^{\prime}}$ are the dyon harmonics ${ }^{[7]}$ and the radial function $U(r) / r_{A_{1}}=R(r)$ satisfies
the equation

$$
r^{2}\left\{\frac{1}{r R(r)} \frac{d^{2}}{d r^{2}}(r R)+2 m(E-V)\right\}=-\frac{\Lambda Y_{e g, l^{\prime}, m^{\prime}}(\theta, \phi)}{Y_{e g, l^{\prime}, m^{\prime}}(\theta, \phi)}=l^{\prime}\left(I^{\prime}+1\right)
$$

with

$$
\Lambda=\frac{1}{\operatorname{Sin} \theta} \frac{\partial}{\partial \theta}\left[\operatorname{Sin} \theta \frac{\partial}{\partial \theta}\right]+\frac{1}{\operatorname{Sin}^{2} \theta}\left(\frac{\partial^{2}}{\partial \phi^{2}}\right)
$$

and

$$
V=-\frac{e_{i} e_{j}}{r_{A_{1}}}+\frac{\left(e_{i} g_{j}\right)^{2}}{2 m r_{A_{1}}^{2}}
$$

Substituting this value of $V$ into equation (3.13), we get 


$$
\frac{d^{2} u}{d r_{A_{1}}^{2}}+\left[2 m E+\frac{2 m e_{i} e_{j}}{r_{A_{1}}}-\frac{l^{\prime}\left(l^{\prime}+1\right)-\left(e_{i} g_{j}\right)^{2}}{r_{A_{1}}^{2}}\right] u=0
$$

which yields the following eigenfunctions for this system

$$
\left.\begin{array}{l}
\psi_{A_{100}}(1)=\frac{1}{\sqrt{\pi a_{0}^{3}}} e^{-r_{A_{1}} / a_{O}} \\
\psi_{A_{200}}(1)=\frac{1}{\sqrt{4 \pi}}\left(\frac{1}{2 a_{0}}\right)^{3 / 2}\left(2-\frac{r_{A_{1}}}{a_{0}}\right) e^{-\frac{r_{A_{1}}}{a_{0}}}
\end{array}\right\}
$$

We can obtain similar wavefunction for the isolated system of dyon -'B' and fermion- ' 2 ' , when choosing the zeroth approximation we must take into account the exchange symmetry of wavefunction due to identical fermions. The two possible spin states of the fermions, the singlet(s) and triplet (t) corresponding to two kinds of the coordinate functions

$$
\left.\begin{array}{l}
\phi_{s}=\left[2\left(1+S^{2}\right)\right]^{-1 / 2}\left\{\psi_{A}(1) \psi_{B}(2)+\psi_{A}(2) \psi_{B}(1)\right\} \\
\phi_{t}=\left[2\left(1-S^{2}\right)\right]^{-1 / 2}\left\{\psi_{A}(1) \psi_{B}(2)-\psi_{A}(2) \psi_{B}(1)\right\}
\end{array}\right\}
$$

where

$$
\left.\begin{array}{l}
\psi_{A}(1)=\frac{1}{\sqrt{\pi a_{0}^{3}}} e^{-r_{A_{1}} / a_{0}} \\
\psi_{A}(2)=\frac{1}{\sqrt{\pi a_{0}^{3}}} e^{-r_{A_{2}} / a_{0}} \\
\psi_{B}(1)=\frac{1}{\sqrt{\pi a_{0}^{3}}} e^{-r_{B_{1}} / a_{0}} \\
\psi_{B}(2)=\frac{1}{\sqrt{\pi a_{0}^{3}}} e^{-r_{B_{2}} / a_{0}}
\end{array}\right\}
$$

$a_{0}$ is the Bohr radius for dyon-fermion system given as ${ }^{[7]}$ 


$$
a_{0}=\frac{\left(e_{i} g_{j}\right)^{2}+1}{m e_{i} e_{j}}
$$

and

$$
S=\int \psi_{A}(1) \psi_{B}(1) d \tau=\frac{1}{\pi a_{0}^{3}} \int e^{-\left(r_{A_{1}}+r_{B_{1}}\right) / a_{0}} d \tau
$$

is the overlap integral of the wavefunction. We can evaluate this integral by the use of elliptical coordinates

$$
\mu=\frac{r_{A_{1}}+r_{B_{1}}}{R}, v=\frac{r_{A_{1}}-r_{B_{1}}}{R}, \phi
$$

where $\phi$ is the angle around the axis connecting two dyons. The volume element in these coordinates has the following form

$$
d \tau=\frac{1}{8} R^{3}\left(\mu^{2}-v^{2}\right) d \mu d v d \phi
$$

Integration is between the limits

$$
1 \leq \mu \leq \infty,-1 \leq v \leq 1,0 \leq \phi \leq 2 \pi
$$

With these coordinates equation (3.21) becomes

$$
S=\int_{1}^{\infty} d \mu \int_{-1}^{1} d v \int_{0}^{2 \pi} d \phi\left[\frac{R^{3}}{8}\left(\mu^{2}-v^{2}\right)\right]=\left[1+\rho+\frac{\rho^{2}}{3}\right] e^{-\rho}
$$

where $\rho=R / a_{0}$.

To evaluate the energy of the system in singlet and triplet spin states, in the first approximation of perturbation theory, we must evaluate, respectively, the integrals

$$
\varepsilon_{s}=\int \phi_{s} \hat{H}_{0} \phi_{s} d \tau \quad \text { and } \quad \varepsilon_{t}=\int \phi_{t} \hat{H}_{0} \phi_{t} d \tau
$$

where subscript $s$ and t stands for singlet and triplet respectively. Substituting equations (3.18) and (3.19) into these expressions, we have

$$
\begin{aligned}
& \Delta E_{S}=\varepsilon_{S}-2 E_{1 S}=\frac{Q+A}{1+S^{2}} \\
& \Delta E_{t}=\varepsilon_{t}-2 E_{1 S}=\frac{Q-A}{1-S^{2}}
\end{aligned}
$$

where $E_{1 \mathrm{~S}}$ are the eigen energies of the isolated system corresponding to the eigen functions (3.19);

$$
\left[\frac{-\hbar^{2}}{2 \mu} \nabla_{1}^{2}-\frac{e^{2}}{r_{A_{1}}}+\frac{(e g)^{2}}{2 m r_{A_{1}^{2}}}\right] \psi_{A}(1)=E_{1 S} \psi_{A}(1)
$$




$$
\begin{gathered}
\text { and } Q=\int \psi_{A}^{2}(1) \psi_{A}^{2}(2)\left[-\frac{e^{2}}{r_{12}}-\frac{e^{2}}{r_{B_{1}}}+\frac{(e g)^{2}}{2 m r_{B_{1}}^{2}}-\frac{e^{2}}{r_{A_{2}}}+\frac{(e g)^{2}}{2 m r_{A_{2}}^{2}}\right] d \tau+\frac{e^{2}+g^{2}}{R} \\
=-\int \psi_{A}^{2}(1)\left(\frac{e^{2}}{r_{B_{1}}}+\frac{(e g)^{2}}{2 m r_{B_{1}}^{2}}\right) d \tau \\
\quad-\int \psi_{B}^{2}(2)\left(\frac{\rho^{2}}{r_{A_{2}}}+\frac{(e g)^{2}}{2 m r_{A_{2}}^{2}}\right) d \tau \\
\quad-\int \psi_{A}^{2}(1) \frac{e^{2}}{r_{12}} \psi_{B}^{2}(2) d \tau+\frac{e^{2}+g^{2}}{R}
\end{gathered}
$$

The first two integrals in this expression determine the average value of the Coulomb interaction between dyon-'B' and fermion-'1' while third and forth integrals determine the interaction between the fermion-'2' and dyon-'A'. Numerically, these two separate sets of integrals are equal to each other.The fifth term determines the Coulomb interaction between fermion 1 and fermion 2. The last term corresponds to the interaction between two dyons.

As such $\mathrm{Q}$ is Coulomb integral which can also be written in the following form from eqn. (3.29); (see appendix A)

$$
\begin{aligned}
Q= & \frac{e^{2}}{a_{0} \rho} e^{-2 \rho}\left[1+\frac{5}{8} \rho-\frac{3}{4} \rho^{2}-\frac{1}{6} \rho^{3}\right]+\frac{(e g)^{2}}{m a_{0}^{2} \rho}\left[1+2 e^{-\rho}(\rho+1)\right. \\
& \left.+\frac{2 e^{-\rho}}{\rho}\left[\frac{\rho-1}{\rho}\right]\right] \\
= & \frac{e^{2}}{a_{0} \rho} e^{-2 \rho}\left[1+\frac{5}{8} \rho-\frac{3}{4} \rho^{2}-\frac{1}{6} \rho^{3}\right] \\
& +\frac{(e g)^{2}}{m a_{0}^{2} \rho^{3}}\left[\rho+2 e^{\left.-\rho\left(\rho+\rho^{2}+\rho^{3}-1\right)\right]}\right.
\end{aligned}
$$

(where $\rho=R / a_{0}$ ).

The interaction energy is determined by the integral

$$
A=\int \psi_{A}(1) \psi_{B}(2)\left[\frac{e^{2}+g^{2}}{R}+\frac{e^{2}}{r_{12}}-\frac{e^{2}}{r_{B_{1}}}+\frac{(e g)^{2}}{2 m r_{B_{1}}^{2}}-\frac{e^{2}}{r_{A_{2}}}\right.
$$




$$
\left.+\frac{(e g)^{2}}{2 m r_{A_{2}}^{2}}\right] \psi_{A}(2) \psi_{B}(1) d \tau_{1} d \tau_{2}
$$

or

$$
\begin{aligned}
A= & \frac{\left(e^{2}+g^{2}\right) S^{2}}{R}+\int \psi_{A}(1) \psi_{B}(2) \frac{e^{2}}{r_{12}} \psi_{A}(2) \psi_{B}(1) d \tau_{1} d \tau_{2} \\
& -S \int \psi_{A}(1) \frac{e^{2}}{r_{B_{1}}} \psi_{B}(1) d \tau_{1}+S \int \psi_{A}(1) \frac{(e g)^{2}}{2 m r_{B_{1}}^{2}} \psi_{B}(1) d \tau_{1} \\
& -S \int \psi_{B}(2) \frac{e^{2}}{r_{A_{2}}} \psi_{A}(2) d \tau_{2}+S \int \psi_{B}(2) \frac{(e g)^{2}}{2 m r_{A_{2}}^{2}} \psi_{A}(2) d \tau_{2}
\end{aligned}
$$

which yields

$$
\begin{aligned}
A= & \frac{\left(e^{2}+g^{2}\right) S^{2}}{R}+\frac{e^{2}}{5 a_{0}}\left[-e^{-2 \rho}\left\{-\frac{25}{8}+\frac{23}{4} \rho+\frac{1}{3} \rho^{2}\right\}\right. \\
+ & \left.\frac{6}{\rho}\left\{S^{2}(C+\log \rho) M^{2} E_{i}(-4 \rho)-2 S M E_{i}(-2 \rho)\right\}\right] \\
& -\frac{2 \rho^{2} e^{-2 \rho}}{3 a_{0}}\left(3+6 \rho+4 \rho^{2}+\rho^{3}\right)+\frac{6(e g)^{2} e^{-\rho}}{m a_{o}^{2}}
\end{aligned}
$$

where $C=0.57722$ is Eular's constant, $E_{i}(x)=\int_{-x}^{\infty} \frac{e^{-\xi}}{\xi} d \xi$ is the exponential integral and $M=e^{\rho}\left(1-\rho+\frac{\rho^{3}}{3}\right)$.

The integrals $\mathrm{Q}$ and $\mathrm{A}$ are functions of the distance between the dyons. The interaction between these two dyons depends mainly on their spin-statistics. In singlet state, when spin of these two dyons are anti-parallel, the interaction energy $\varepsilon_{s}$ increases forming a bound state while in triplet state, when spin of these particles are parallel, the energy $\varepsilon_{t}$ increases monotonically showing a possible repulsion between the dyons. These predictions can be analyzed with the help of equation (3.18), where coordinate function corresponding to triplet state has nodes in plane perpendicular to the axis connecting two dyons and bisecting it. On the other hand, function (3.18) corresponding to singlet spin state has its largest value in that plane. Thus in the singlet spin state, for $\rho$ $\sim 1$, there is a large probability that fermions occupy the position between the dyons. The Coulomb attraction between the fermions and dyons leads to a bound state. However, the probability of forming this bound state will be very small since the repulsive force 
between dyons is large and the distance of closest approach is much smaller than $a_{O}$. For distance $R\left\langle a_{o}\right.$ fermions can not lie in between dyons, not even in singlet state and repulsion occurs. In the triplet spin state the probability is small of finding the fermions between the dyons for all distances which are very large and we have a repulsion which decreases exponentially with the distances.

The different properties of the singlet and triplet states are quantitatively determined by the values of the exchange integral A. From equation (3.32) of this integral, it follows that its integrand is non-zero only at those points in space where the product $\psi_{A}(1) \psi_{B}(1)$ and $\psi_{A}(2) \psi_{B}(2)$ are non-vanishing i.e. in the region where the fermion wave functions of the two fermions overlap.

Now, we can evaluate $E_{1 S}$ in the following form

$$
E_{1 S}=\int \psi_{A}(1)\left[-\frac{\hbar^{2}}{2 \mu}-\frac{e^{2}}{r_{A_{1}}}+\frac{(e g)^{2}}{2 m r_{A_{1}}^{2}}\right] \psi_{A}(1) d \tau
$$

Solving it with the help of elliptical coordinates i.e. eqn. (3.22), we get

$$
E_{1 S}=-\frac{e^{2}}{2 a_{0}}+\frac{(e g)^{2}}{2 m \rho a_{0}^{2}}\left[\left(1+e^{-2 \rho}\right)+\left(1-\rho^{2}\right)\left(2+e^{-2 \rho}\right)\right]
$$

Substituting the value of $E_{1 S}$ from eqn. (3.34) to eqn. (3.27), we get

$$
\begin{aligned}
\varepsilon_{S}= & -\frac{2 e^{2}}{a_{0}}+\frac{(e g)^{2}}{m \rho a_{0}}\left[3-\left\{\left(2-\rho^{2}\right)\left(2 \rho-e^{-2 \rho}\right)\right\}\right] \\
& +\frac{\frac{9(e g)^{2}}{\rho m a_{0}^{2}} e^{\rho}(14 \rho+1)-\frac{e^{2}}{40 a_{0} \rho}\left[45+1509 \rho+1446 \rho^{2}+324 \rho^{3}-R\right]}{\left(9+18 \rho+15 \rho^{2}+6 \rho^{3}+\rho^{4}+9 e^{2 \rho}\right)}
\end{aligned}
$$

where $R=\frac{6}{\rho}\left\{S^{2}(C+\log \rho)+M^{2} E_{i}(-4 \rho)-2 S M E_{i}(-2 \rho)\right\}$

Similarly, we obtain

$$
\begin{aligned}
\varepsilon_{t} & =-\frac{2 e^{2}}{a_{0}}+\frac{(e g)^{2}}{m \rho a_{0}}\left[3-\left\{\left(2-\rho^{2}\right)\left(2 \rho-e^{-2 \rho}\right)\right\}\right] \\
& +\frac{\frac{9(e g)^{2}}{\rho m a_{0}^{2}}(2 \rho+1)+\frac{e^{2}}{40 a_{0} \rho}\left[755+2079 \rho+1089 \rho^{2}+204 \rho^{3}-R\right]}{\left(9 e^{2 \rho}-\rho^{4}-6 \rho^{3}-15 \rho^{2}-18 \rho-9\right)}
\end{aligned}
$$

and the splitting in singlet and triplet states are obtained as 


$$
\begin{aligned}
\Delta E_{S} & =\varepsilon_{S}-2 E_{1 S}=\frac{Q+A}{1+S^{2}} \\
& =\frac{\frac{9(e g)^{2} e^{\rho}}{\rho m a_{0}^{2}}(2 \rho+1)+\frac{e^{2}}{40 a_{0} \rho}\left[45+1509 \rho+1446 \rho^{2}+324 \rho^{3}+R\right]}{\left(9 e^{2 \rho}+\rho^{4}+6 \rho^{3}+15 \rho^{2}+18 \rho+9\right)}
\end{aligned}
$$

For triplet state, we obtained the following expression

$$
\begin{aligned}
& \Delta E_{t}=\varepsilon_{t}-2 E_{1 S}=\frac{Q-A}{1-S^{2}} \\
& =\frac{\frac{9(e g)^{2} e^{\rho}}{\rho m a_{0}^{2}}(2 \rho+1)+\frac{e^{2}}{40 a_{0} \rho}\left[755+2079 \rho+1089 \rho^{2}+204 \rho^{3}-R\right]}{\left[9 e^{2 \rho}-\rho^{4}-6 \rho^{3}-15 \rho^{2}-18 \rho-9\right]}
\end{aligned}
$$

Fig. 1 Indices ' $A$ ' and ' $B$ ' denote dyons and indices ' 1 ' and ' 2 ' denote fermions

\section{CONCLUSIONS}

Equation (2.5) is the rotational as well as gauge symmetric angular momentum of dyons and equation (2.7) is the Hamiltonian for a dyon moving in the generalized 
electro- magnetic field of another dyon. This Hamiltonian is modified when we take in to account bound state of two fermions and two dyons as shown in Fig. (1). The modified Hamiltonian for this system is given by equation (3.2) and interaction potential energy is given by equation (3.3). The energy of this system has been obtained in the form of equations (3.37) and (3.38) for singlet and triplet state respectively depending on the spin-statistics relations of the fermions and dyons involved in the formation of bound states. These energy values are quite different from the energy eigen values of the

Hydrogen molecule ${ }^{[16]}$ due to the presence of magnetic charge on dyon. However, these energy eigen values reduce to ordinary Hydrogen molecule energy eigen values in the absence of magnetic charge. Based on this formulation, we can also consider muons (negatively charged) in place of fermion. The muonic bound state energy will also be given by equations (3.37) and (3.38) with the Bohr radius of the system modified by

$a_{\mu}=\left[\frac{\left(e_{i} g_{j}\right)^{2}+1}{M_{\mu} e_{i} e_{j}}\right]=\frac{a_{o}}{207}$ as muon is 207 times heavier than the electron. If the muon bound state is possible it will be very unstable due to the high repulsive force between moun and electric charge of dyon. This decay process may lead to certain new insight in to physics at incredibly short distances because muon is very unstable with an average life time of $10^{-6}$ seconds and decays in to an electron and two neutrinos.

\section{Appendix-A}

Let us consider the quantitative value of the integral Q. Substituting the explicit form (3.19) into (3.29) and using the fact that first two integrals are same, we find;

$$
Q_{1}=-\frac{2 e^{2}}{\pi a_{0}^{3}} \int \frac{e^{-2 A_{1} / a_{0}}}{r_{B_{1}}} d \tau+\frac{(e g)^{2}}{m \pi a_{0}^{3}} \int \frac{e^{-2 r_{1} / a_{0}}}{r_{B_{1}^{2}}} d \tau
$$

The first part of this equation may be calculated easily by using elliptical coordinates. We can write the second part of above equation in the following form;

$$
\begin{aligned}
\frac{(e g)^{2}}{m \pi a_{0}^{3}} \int \frac{e^{-2 r_{A_{1}} / a_{0}}}{r_{B_{1}^{2}}} d \tau & =\frac{(e g)^{2}}{m \pi a_{0}^{3}} \cdot \frac{4 R^{3}}{8 R^{2}}\left[\int_{+1}^{\infty} \int_{-1}^{+12 \pi} \int_{0} \frac{\left(\left(\mu_{1}^{2}-v_{1}^{2}\right)\right)}{\left(\mu_{1}-v_{1}\right)^{2}} e^{-\rho\left(\mu_{1}+v_{1}\right)} d \mu_{1} d v_{1} d \varphi_{1}\right. \\
& +\int_{+1}^{\infty} \int_{-1}^{+12 \pi} \int_{0} \frac{\left(\mu_{2}^{2}-v_{2}^{2}\right)}{\left(\mu_{2}+v_{2}\right)} e^{-\rho\left(\mu_{2}-v_{2}\right)} d \mu_{2} d v_{2} d \phi_{2}
\end{aligned}
$$


where $\mu, v$ and $\phi$ are elliptical coordinates defined as equation (3.22). Equation (A-2) yields the following value by using binomial expansion and retaining the terms up to first order. Therefore

$$
\frac{(e g)^{2} R}{m \pi a_{0}^{3}} \int \frac{e^{-2 r_{A_{1}} / a_{0}}}{r_{B_{1}^{2}}} d \tau=\frac{(e g)^{2} R}{m a_{0}^{3} \rho^{2}}\left[\left\{1+2 e^{-\rho}(1+\rho)-2 e^{-\rho}(1-\rho)+2 \int_{+1}^{\infty} \frac{e^{-\rho \mu}}{\mu} d \mu\right\}\right]
$$

After solving equation (A-3), we get

$$
\begin{gathered}
\frac{(e g)^{2}}{m \pi a_{0}^{3}} \int \frac{e^{-{ }^{2 r_{A_{1}} / a_{0}}} d \tau}{r_{B_{1}^{2}}}=\frac{(e g)^{2} R}{m \pi a_{0}^{3}}\left[\int_{+1}^{\infty} \int_{-1}^{+1}\left(1+\frac{2 v_{1}}{\mu_{1}}\right) e^{-\rho\left(\mu_{1+v_{1}}\right) d \mu_{1} d v_{1}}\right. \\
\left.+2 \int_{+1}^{\infty} \frac{e^{-\rho \mu}}{\mu} d \mu\right]
\end{gathered}
$$

We now evaluate the integral

$$
K=\int_{+1}^{\infty} \frac{e^{-\rho \mu}}{\mu} d \mu
$$

Solving the above exponential integral by error function method, we get

$$
K=\frac{e^{-\rho}}{\rho}\left[1-\frac{1}{\rho}\right]
$$

Finally, we obtain

$$
\begin{aligned}
Q & =\frac{e^{2}}{a_{o} \rho} e^{-2 \rho}\left[1+\frac{5}{8} \rho-\frac{3}{4} \rho^{2}-\frac{1}{6} \rho^{3}\right] \\
& +\frac{(e g)^{2}}{m a_{0}^{2} \rho^{3}}\left[\rho+2 e^{-\rho}\left(\rho+\rho^{2}+\rho^{3}-1\right)\right]
\end{aligned}
$$

\section{REFERENCES}

1. P.A.M. Dirac; Proc. R. Soc. London A 133 (1931) 60.

2. Y.M. Cho; Phys. Rev. D 21 (1980) 1080.

M.N. Saha; Ind. J. Phys. 10 (1936) 145.

M. Peshkin; Ann Phys. 113 (1978) 122.

3. C.J. Callon; Jr. Phys. Rev. D 26 (1982) 2058 .

Phys.Rev. D 25 (1982) 2141.

V. Ruba kov; Nucl. Phys. B203 (1982) 311. 
4. G't Hooft; Nucl. Phys. B 153 (1979) 14.

Nucl. Phys. B 190 (1981) 45.

5. S. Mandelstam; Phys. Rep. 23 (1976) 245.

Phys. Rev. D 9 (1979) 249.

6. C. Dokos and T. Tomaras; Phys. Rev. D 21 (1980) 2940.

7. A.S. Goldhaber; Phys. Rev. Lett. $\underline{36}$ (1976) 1122.

8. G't Hooft; Nucl. Phys. B 79 (1974) 276.

9. A.M. Polyakov; JETP Lett. 20 (1974) 174.

10. E. Witten; Phys. Lett. B 87 (1987) 283.

11. B.S. Rajput and D.C. Joshi; Had. Jour. 4 (1981) 1805.

B.S. Rajput and Om Prakash; Ind. J. Phys. A 53 (1979) 274.

B.S. Rajput and D.S. Bhakuni; Lett. Nuovo Cim. 34 (1982) 509.

B.S. Rajput; J.Math. Phys. 25 (1984) 351.

B.S. Rajput; Lett. Nuovo Cim. 35 (1982) 205.

12. P.C. Pant, V.P. Pandey and B.S. Rajput; Il. Nuovo Cim. A 110 (1997) 829.

13. P.C. Pant, V.P. Pandey and B.S. Rajput; Il. Nuovo Cim. A 110 (1997)1421.

14. P.C. Pant, V.P. Pandey and B.S. Rajput; Ind. J. Phys. (Communicated).

15. B.S. Rajput and V.P. Pandey; Nuovo Cim. B 111 (1996) 275.

16. L. Pauling ; Chem. Rev. $\underline{5}$ (1928) 173. 